\title{
Dynamique des peuplements ligneux dans le Parc National de Manda, au sud du Tchad
}

\author{
Guiguindibaye MADJIMBE $^{1 *}$, Goy SARADOUM ${ }^{2}$, Touroumgaye GOALBAYE ${ }^{1}$, Esaïe \\ WAYA $^{1}$ et Jonathan POUNAKOUMNA ${ }^{1}$ \\ ${ }^{1}$ Université de Sarh, Institut Universitaire des Sciences Agronomiques et de l'Environnement (IUSAE), BP 105 Sarh, Tchad. \\ ${ }^{2}$ Université de Sarh. Faculté des Sciences et Techniques (FAST). BP 105 Sarh, Tchad. \\ *Auteur correspondant, E-mail: madjimbe1@yahoo.fr, Tél: +23566393529 / 91946807
}

Mots clés : Peuplement ligneux, Caractéristiques structurales, régénération, Tchad

Key words: Woody stock, Structural features, regeneration, Chad

Publication date 31/10/2019, http://www.m.elewa.org/JAPS

\section{RÉSUMÉ}

La présente étude a pour but d'étude la dynamique des peuplements ligneux dans le Parc National de Manda. Elle vise à déterminer les caractéristiques structurales, la composition spécifique et la régénération des peuplements ligneux. Quatre sites ont été implantés depuis 2012. Nous avons procédé à la caractérisation des peuplements ligneux par des relevés dendrométriques. La flore ligneuse dans l'ensemble des sites est riche de 87 espèces reparties en 56 genres et 22 familles. Les sites 1 et 2 ont des effectifs plus élevés en 2019 comparativement en 2014 et en 2012. Une forte régénération s'est effectuée durant l'année 2019. Contrairement aux sites, 1 et 2, les sites 3 et 4 présentent pour l'année 2019 une forte baisse des effectifs de jeunes plants. Ces jeunes plants sont très sensibles non seulement à la disponibilité des ressources en eau pour franchir la saison sèche mais aussi à l'intensité et à la fréquence de passage des feux. Les résultats ont permis de noter que la densité moyenne entre 2012 et 2014 s'est accrue et à partir de 2014 elle s'est stabilisée. La surface terrière par hectare est, en moyenne de $10,12 \mathrm{~m}^{2} /$ ha pour $2012 ; 11,16 \mathrm{~m}^{2} /$ ha pour l'année 2014 et de 11, 65 $\mathrm{m}^{2}$ /ha pour l'année 2019. Pour l'ensemble des sites expérimentaux, on évalue le volume moyen de bois sur pieds à 12,31 $\mathrm{m}^{3} /$ ha en 2012 , à $12,46 \mathrm{~m}^{3} /$ ha en 2014 et $12,08 \mathrm{~m}^{3} /$ ha en 2019. Ces valeurs indiquent que les variations de volume moyen de bois sur pieds sont très faibles pour les sites pris dans leur ensemble. Les résultats obtenus, montrent aussi que 8 espèces se situent dans un processus d'évolution régressive et 4 autres dans un processus d'évolution progressive. La structure des peuplements ligneux n'a pas beaucoup évolué en 8 ans mais, elle apparaît fortement tributaire de la topographie et de la pression anthropique.

\section{ABSTRACT \\ Dynamic of the woody populations in the National Park of Manda, to the south of Chad}

The purpose of this study is to study the dynamics of woody stands in the Manda National Park. It aims to determine the structure, specific composition and regeneration of woody stands. Four sites have been established since 2012. We have characterized the woody stands by dendrometric surveys. The woody flora in all sites is rich in 87 species divided into 56 genera and 22 families. Sites 1 and 2 have higher numbers in 2019 compared to 2014 and 2012. Strong regeneration took place in 2019. Unlike sites 1 and 2, sites 3 and 4 present for the year 2019 a sharp decline in the number of young plants; these seedlings are very sensitive not only to the availability of water resources to cross the dry season but also to the 
intensity and frequency of fire. The results showed that the average density between 2012 and 2014 increased and from 2014 it stabilized. The basal area per hectare is, on average, $10.12 \mathrm{~m} 2$ / ha for 2012; $11.16 \mathrm{~m} 2$ / ha for the year 2014 and 11, $65 \mathrm{~m} 2$ / ha for the year 2019. For all the experimental sites, the average volume of standing timber is estimated at $12.31 \mathrm{~m}^{3}$ / ha in 2012, $12.46 \mathrm{~m}^{3}$ / ha in 2014 and $12.08 \mathrm{~m}^{3}$ / ha in 2019. These values indicate that variations in average volume of standing timber are very low for sites taken as a whole. The results obtained also show that 8 species are in a process of regressive evolution and 10 in a process of gradual evolution. The structure of woody stands has not changed much in 8 years, but it appears highly dependent on topography and anthropogenic pressure.

\section{INTRODUCTION}

Le processus de dégradation constaté au niveau des aires protégées affecte la qualité de la vie des communautés rurales (Minda et al., 2015). Le réseau des aires protégées au Tchad couvre près de $10,2 \%$ de la surface du pays et reste globalement représentatif de toute la diversité des écosystèmes de la région (Ballah, 2008). Ces aires protégées subissent une forte dégradation de leurs ressources végétales en raison de la péjoration climatique et de la pression anthropique (Badji et al., 2014). Le prélèvement sélectif de certaines espèces de bois d'œuvre de qualité ou des arbres fourragers menace dangereusement les ressources phytogénétiques du pays (Saradoum, 2012). Parmi les espèces ainsi menacées, se trouvent Pterocarpus erinaceus espèce bien inféodée aux zones tropicales sahéliennes arides et soudaniennes semi-aride (Adjonou et al., 2010), Prosopis africana, dont le tronc peut être utilisé comme support des greniers durant une dizaine d'années (Madjimbe et al., 2018) et Afzelia africana, plante fourragère très appétées par le bétail (Sarr et al., 2013). Le Parc National de Manda (PNM), site d'étude à l'image des autres aires protégées du pays, a subi particulièrement une réduction des boisements, d'une coupe intensive et incontrôlée du bois frais et d'une pression accrue sur les ressources pastorales (Photo 1)

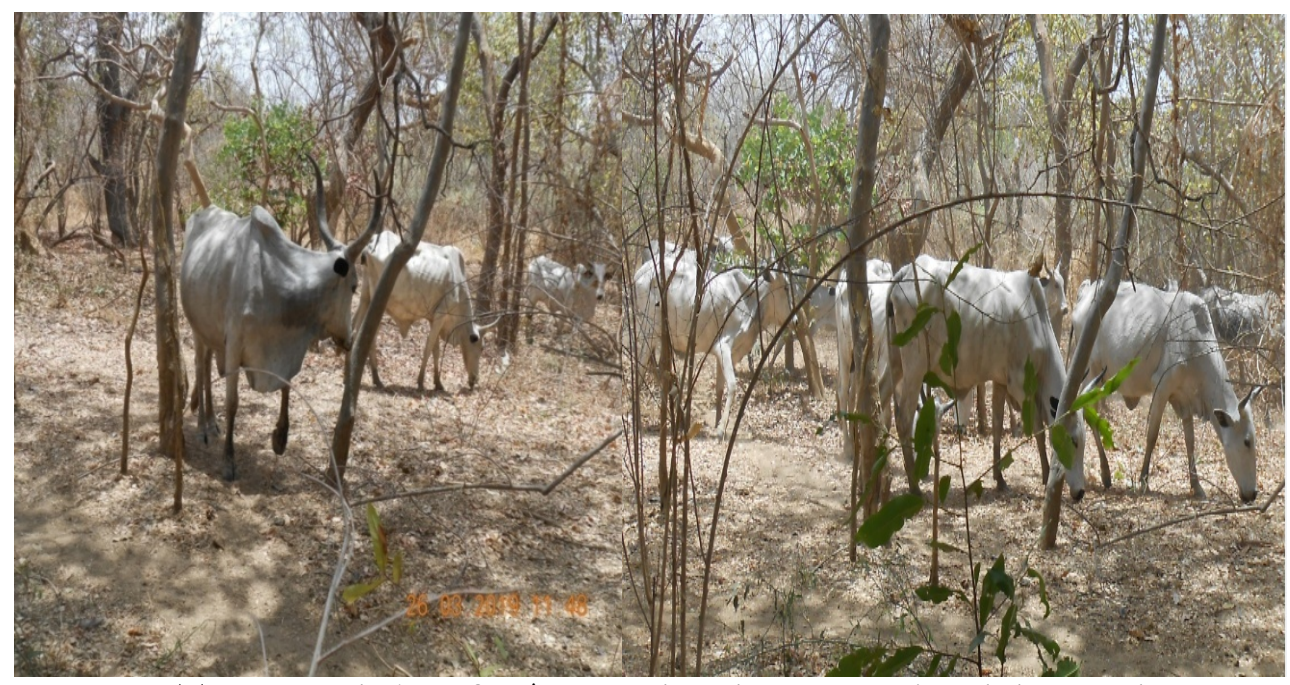

Photo 1 : Troupeau de bœufs pâturant dans le Parc National de Manda.

Photo G. Madjimbe

Le pays ne dispose pas d'informations scientifiques et techniques permettant d'orienter sa sylviculture. Par ailleurs, très peu d'études sont disponibles pour connaître l'état actuel des peuplements naturels du PNM afin d'établir des stratégies adéquates 
d'aménagement. Les quelques références disponibles concernent généralement la description des formations naturelles des zones soudaniennes (forêts claires, savanes boisées et arborées.), la typologie et la cartographie, l'inventaire floristique et la phytosociologie (Kolmagne, 2000 ; Ouya, 2005; Ballah, 2008 ; Chardonnet et Boulanodji, 2010; Sardoum, 2012). Ce qui précède justifie l'urgence de cette étude sur la dynamique des peuplements

\section{MATÉRIEL ET MÉTHODES}

\subsection{Présentation de la zone d'étude : Le} Parc National de Manda (PNM) est situé à 25 $\mathrm{km}$ au nord-ouest de Sarh chef-lieu du Département du Barh Kôh, Province du Moyen Chari. Il est compris entre $9^{\circ} 20^{\prime}$ et $9^{\circ} 35^{\prime}$ de latitude Nord et $17^{\circ} 45^{\prime}$ et $18^{\circ} 20^{\prime}$ de longitude Est. Son altitude varie de $344 \mathrm{~m}$ à $691 \mathrm{~m}$. Il est limité au sud par le Barh Sara, à l'ouest par la route nationale Sarh-Ndjamena, à l'est par le fleuve Chari et au nord par la piste du croisement Sarh-Ndjamena vers Waïn (figure 1). Cette entité forestière de 114000 ha est créée afin de protéger la faune et la flore de la zone soudanienne et l'article 2 de sa création stipule que « ce Parc National est constitué en vue de la propagation, la protection et la conservation de la vie animale sauvage et de la végétation sauvage dans un intérêt scientifique et éducatif au profit, à l'avantage et pour la récréation du public »(Décret $\left.n^{\circ} 56,1965\right)$. La zone est caractérisée par un climat de type soudanien avec une pluviométrie moyenne annuelle de 1061,41 mm. Les températures annuelles sont de l'ordre de $21^{\circ} \mathrm{C}$ à $28^{\circ} \mathrm{C}$ avec une humidité relative selon les mois de 32 à 85\% (ASECNA de Sarh, 2018). Les sols sont variés, on rencontre les principaux types suivants: les sols d'érosion sur roches acides issus de l'érosion qui constituent le Mont Niellim ; les sesquioxydes à tâches et concrétions ferrugineuses et cuirasses qui se rencontrent sur les dalles latéritiques dans la zone de Niellim ; les vertisols dans certaines zones inondables entre Koutou et Niellim; les sols ferralitiques qui affleurent largement dans naturels ligneux dans le PNM. Cette étude vise à déterminer par un suivi spatio-temporel, les tendances évolutives de la végétation naturelle ligneuse sous l'effet des mesures d'aménagement. L'évaluation des caractéristiques structurales de l'espèce et de son potentiel de régénération constituent des indicateurs écologiques essentiels pour mesurer le niveau d'utilisation, la dynamique et la santé de la ressource dans son biotope naturel.

les zones de Koutou et de Djoli ; les sols ferrugineux tropicaux au centre du parc, de Nguéré à Koutou et les sols hydromorphes caractéristiques des sols du Sud (Pias, 1964). Le PNM est desservie par un réseau hydrographique dans sa partie orientale par le fleuve Chari et au Sud par le Barh Sara. Deux des trois principaux affluents du Chari ont leurs confluents dans le PNM : le Bahr Sara et le Bahr Salamat. En outre, il est identifié 13 mares temporaires et 23 mares permanentes dans le parc (Tchago, 1999). La végétation est formée de savanes diverses, de forêts claires, et de galeries forestières. La diversité de la faune sauvage est sous-estimée. Seuls les vertébrés peuvent être considérés comme moyennement étudiés. Au sujet de la faune, UICN (2008) signalaient une dizaine de famille de vertébrés sauvage. La zone périphérique entourant le PNM regroupe 85592 habitant répartis dans quatre cantons: Balimba au sud, Kokaga à l'est, Djoli à l'ouest et Niellim au Nord (Saradoum, 2012). La population périphérique du PNM est essentiellement constituée d'agriculteurs. Les activités de pêche concernent principalement la population riveraine des cours d'eau et des pêcheurs. Les terroirs riverains de parc constituent également une zone d'accueil pour des pasteurs de plus en plus nombreux. Selon l'enquête menée par Ballah (2008), le bétail sédentaire est estimé à 28843 têtes de bovins et 13789 de caprins. Le taux annuel du cheptel de ruminants est estimé par la Direction de l'Elevage et des Ressources Animales (DERA) à $2,4 \%$ par an. 


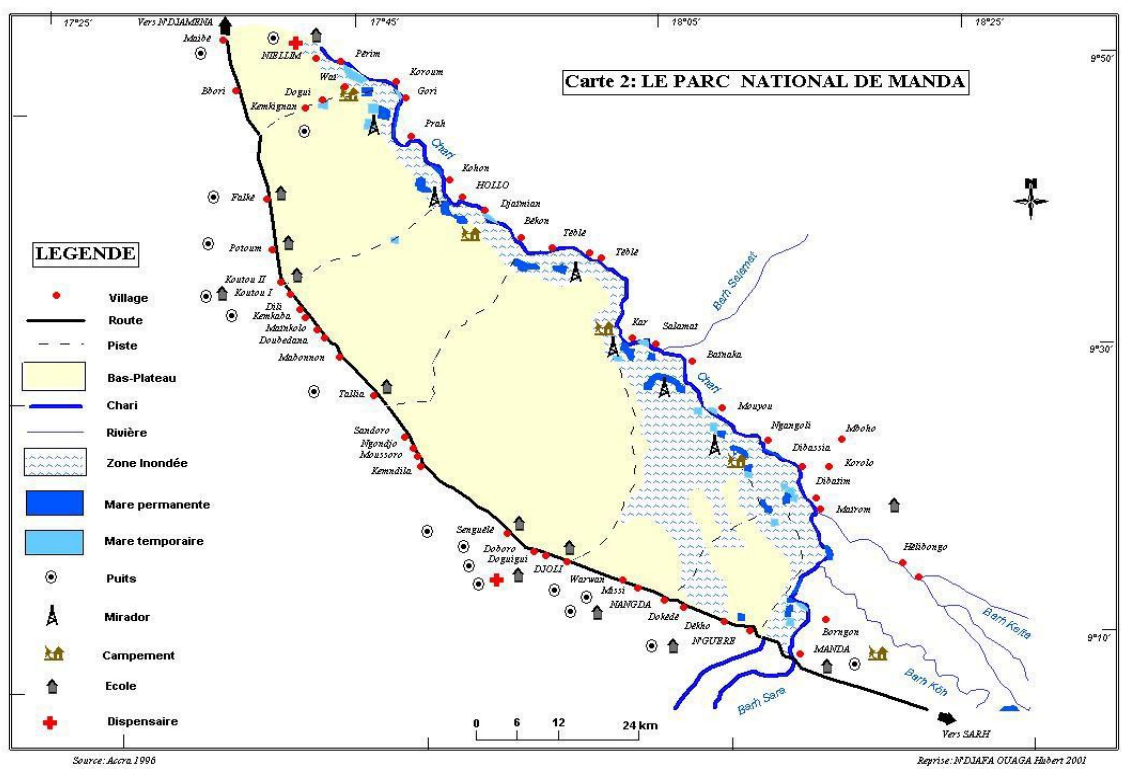

Figure 1 : Carte du Parc National de Manda (ACCRA, 1996)

2.2 Choix des sites d'étude : Le choix des sites s'est fondé sur la représentativité des unités de végétation naturelle, les caractéristiques morpho-pédologiques et l'accessibilité. A cet effet, le support cartographique a été utilisé. Des sorties de reconnaissances ont été également effectuées afin d'orienter ce choix. Après leur implantation, les coordonnées géographiques des centres des sites ont été relevées au GPS. Le tableau I suivant présente les formations végétales abritant les sites et les coordonnées géographiques de ces derniers.

Tableau 1 : Types de formations végétales et coordonnées géographiques des sites

\begin{tabular}{|c|c|c|c|c|}
\hline \multicolumn{5}{|c|}{ Parc National de Manda } \\
\hline Sites & $\mathbf{1}$ & $\mathbf{2}$ & $\mathbf{3}$ & $\mathbf{4}$ \\
\hline $\begin{array}{c}\text { Type de } \\
\text { formations } \\
\text { végétales }\end{array}$ & $\begin{array}{c}\text { Savane arborée } \\
\text { claire }\end{array}$ & Savane arbustive & $\begin{array}{c}\text { Savane arborée claire } \\
\text { sur cuirasse affleurant }\end{array}$ & Forêt galerie \\
\hline $\begin{array}{c}\text { Coordonnées } \\
\text { géographiques } \\
\text { des sites }\end{array}$ & $9^{\circ} 38^{\prime} 31^{\prime \prime} \mathrm{N}$ & $9^{\circ} 39^{\prime} 53^{\prime \prime} \mathrm{N}$ & $9^{\circ} 39^{\prime} 37^{\prime \prime} \mathrm{N}$ & $9^{\circ} 39^{\prime} 50^{\prime \prime} \mathrm{N}$ \\
$17^{\circ} 32^{\circ} 14^{\prime \prime} \mathrm{E}$ & $17^{\circ} 47^{\prime} 47^{\prime \prime} 7^{\prime} \mathrm{E}$ & $17^{\circ} 48^{\prime} 34^{\prime} \mathrm{E}$ \\
\hline
\end{tabular}

2.3 Dispositif de suivi : Le dispositif de suivi dans le PNM est constitué de quatre sites mis en place en 2012. Chaque site se compose de quatre parcelles de $2500 \mathrm{~m}^{2}$ (50 m x $\left.50 \mathrm{~m}\right)$. Pour les besoins des relevés, les parcelles sont subdivisées chacune en 100 placeaux de $25 \mathrm{~m}^{2}$ ( $5 \mathrm{~m} \times 5 \mathrm{~m}$ ). Les placeaux constituent des unités d'énumération. Afin d'assurer la pérennité des sites, des bornes ont été fixés à tous les $10 \mathrm{~m}$.

2.4 Collecte de données : La collecte de données a concerné uniquement la végétation ligneuse. Des inventaires de la végétation ligneuse ont été réalisés en 2012, 2014 et 2019. Cependant, la série de donnée est incomplète pour certains sites où les feux de brousse tardifs sont passés avant l'équipe de relevé. La méthode d'inventaire adoptée est de type intégral. Plusieurs variables portant sur la végétation ont été notées sur chaque individu: le nom scientifique, les numéros de brin et de souche, la circonférence à la base (environ 10 
$\mathrm{cm}$ du sol) et à $1,30 \mathrm{~m}$, ainsi que la hauteur totale de l'individu.

\subsubsection{Les numéros de brin et de souche :} Dans le cadre de cet inventaire, la souche désigne le pied. Elle peut porter une ou plusieurs tiges appelées brin. Avec ces variables les calculs de densité seront possibles car le comptage concerne tous les individus du milieu indépendamment de leur dimension.

\subsubsection{La circonférence à la base (environ} $10 \mathrm{~cm}$ ) et à $1,30 \mathrm{~m}$ du sol : La circonférence entre dans l'estimation de plusieurs paramètres caractéristiques de la végétation: structure, surface terrière et volume de bois sur pieds. Il importe de revenir sur la surface terrière qui est la somme des sections transversales des arbres du peuplement et qui est un indicateur fiable de l'évolution de la végétation ligneuse (photo 2).

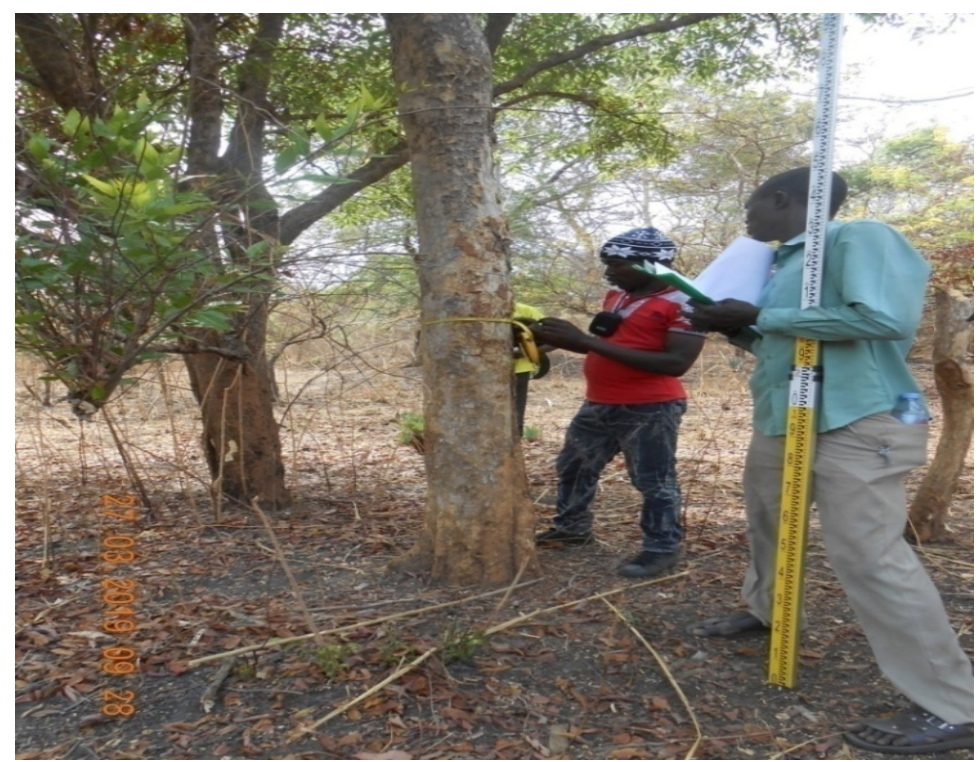

Photo 2: Mesure de circonférence et de la hauteur totale dans le PNM Photo G. Madjimbe

2.4.3 La hauteur totale de l'individu : $\mathrm{La}$ hauteur totale se mesure à l'aide de mire. Cette variable entre dans le calcul des volumes de bois sur pieds. En outre, elle permet de caractériser la structure verticale du peuplement.

2.5 La Saisie, le traitement et l'analyse des données : Les données collectées à l'aide de fiches d'inventaire ont été saisies. Le traitement statistique des données a été fait grâce au logiciel Microsoft Excel 2007. Nous avons calculé la richesse spécifique (S) et exprimé la diversité au moyen de l'indice de Shannon H'. C'est un indicateur de la densité spécifique d'un peuplement et permet de mesurer la biodiversité. Cet indice permet aussi de quantifier l'hétérogénéité de la biodiversité d'un milieu d'étude et donc d'observer une évolution au cours du temps. Il se calcule sur la base de la formule suivante :

$$
\mathrm{H}^{\prime}=-\sum_{i=1}^{s} p i \operatorname{lnpi}
$$

$i$ : une espèce du milieu d'étude; $p i$ : abondance relative de l'espèce $i$ dans l'échantillon

$$
p i=n i / N
$$

où $n i=$ effectif des populations $i$ et $N=$ somme des effectifs de $S$ espèces constituant le peuplement. Les paramètres structuraux retenus pour caractériser les peuplements sont la circonférence à la base, la surface terrière, la densité et les volumes de bois sur pied. La circonférence à la base et à $1,30 \mathrm{~m}$ du sol se mesure à l'aide de ruban. La surface terrière, appelée aussi recouvrement basal, est la somme des surfaces des troncs des arbres à 1,30 m. La surface terrière peut être estimée en considérant 
que les sections des tiges sont circulaires. Elle s'exprime par unité de surface $\left(\mathrm{m}^{2}\right)$ et se calcule à l'aide de la formule suivante :

$$
S t=\sum_{k=1}^{n} \frac{c^{2}}{4 \pi}
$$

$\mathrm{St}=$ Surface terrière exprimée en $\mathrm{m}^{2} / \mathrm{ha}$; $\mathrm{C}=$ Circonférence à $1,30 \mathrm{~m}$ du sol des individus, $\pi=3,14$

\section{RÉSULTATS}

3.1. Les communautés végétales: Les tableaux 2 et 3 présentent le nombre total de famille de ligneux rencontrée, le taux de
La densité d'un peuplement ligneux exprime le nombre total d'arbres par unité de surface (hectare). Le volume de bois sur pied se calcule sur la base de la formule approximative très simple de Denzin (1929), avec une erreur ne dépassant pas $20 \%$ :

$$
V=d^{2} / 1000
$$

où $V=$ Volume bois fort tige en $\mathrm{m}^{3}$ et $\mathrm{d}=$ diamètre à $1,30 \mathrm{~m}$ en $\mathrm{cm}$

recouvrement moyen et la hauteur moyenne par site (1 ou 2) et selon l'année.

Tableau 2 : Evolution de nombre total de familles ligneuses, le taux de recouvrement moyen et la hauteur moyenne par site (1 ou 2) et selon l'année (Parc National de Manda)

\begin{tabular}{|c|c|c|c|c|c|c|}
\hline & \multicolumn{3}{|c|}{ Site 1 } & \multicolumn{3}{c|}{ Site 2 } \\
\cline { 2 - 7 } & $\mathbf{2 0 1 2}$ & $\mathbf{2 0 1 4}$ & $\mathbf{2 0 1 9}$ & $\mathbf{2 0 1 2}$ & $\mathbf{2 0 1 4}$ & $\mathbf{2 0 1 9}$ \\
\hline Nombre total de familles ligneuses & 18 & 24 & 20 & 13 & 20 & 18 \\
\hline Taux de recouvrement moyen (\%) & 56 & 42 & 41 & 63,58 & 65,5 & 68,8 \\
\hline Hauteur moyenne (m) & 12 & 10,7 & 11 & 4,6 & 5,82 & 6 \\
\hline
\end{tabular}

Tableau 3 : Evolution de nombre total de familles ligneuses, le taux de recouvrement moyen et la hauteur moyenne par site (3 ou 4) et selon l'année (Parc National de Manda)

\begin{tabular}{|l|c|c|c|c|c|c|}
\hline & \multicolumn{3}{|c|}{ Site 3 } & \multicolumn{3}{c|}{ Site 4 } \\
\cline { 2 - 7 } & $\mathbf{2 0 1 2}$ & $\mathbf{2 0 1 4}$ & $\mathbf{2 0 1 9}$ & $\mathbf{2 0 1 2}$ & $\mathbf{2 0 1 4}$ & $\mathbf{2 0 1 9}$ \\
\hline Nombre total de familles ligneuses & 17 & 20 & 15 & 22 & 20 & 22 \\
\hline Taux de recouvrement moyen (\%) & 57,5 & 56 & 49 & 86,5 & 87,5 & 87 \\
\hline Hauteur moyenne (m) & 11,8 & 11,7 & 10,5 & 20 & 21 & 25 \\
\hline
\end{tabular}

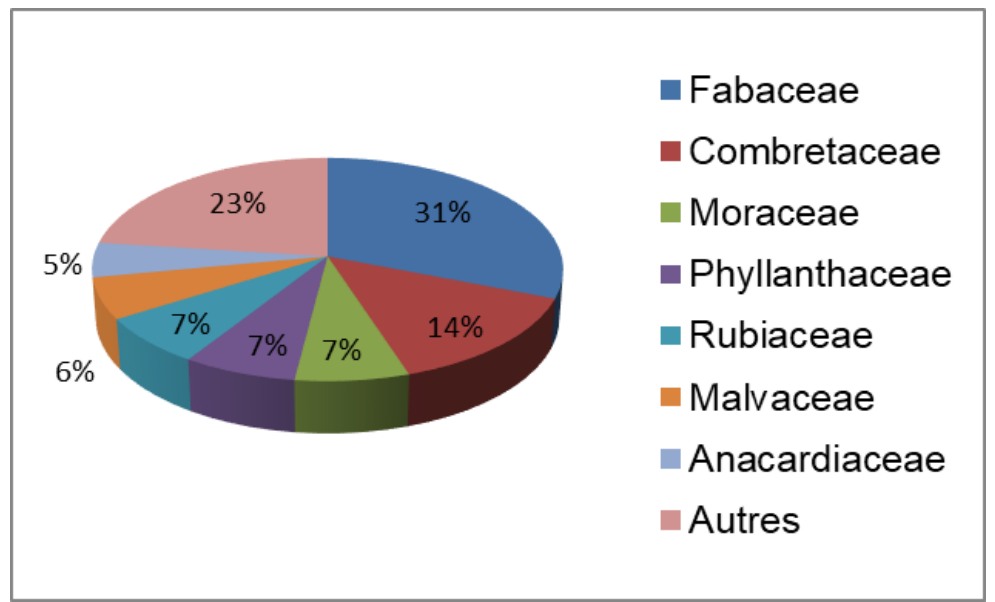

Figure 2: Répartition du pourcentage total d'espèces par familles de ligneux (Parc National de Manda) 
La figure 2 consigne la répartition des proportions totales d'espèces par famille de ligneux recensée dans les sites expérimentaux. Les Fabaceae et les Combretaceae dominent les peuplements avec respectivement $31 \%$ et $14 \%$ du nombre total d'espèces rencontrées. Le Tableau 4 présente l'évolution du nombre total d'espèces ligneuses par site et selon l'année dans les 4 sites d'étude.

Tableau 4: Evolution du nombre total des espèces ligneuses par site et selon l'année (Parc National de Manda)

\begin{tabular}{|l|c|c|c|c|c|c|}
\hline \multirow{2}{*}{} & \multicolumn{3}{|c|}{ Site 1 } & \multicolumn{3}{c|}{ Site 2 } \\
\cline { 2 - 7 } & 2012 & $\mathbf{2 0 1 4}$ & $\mathbf{2 0 1 9}$ & $\mathbf{2 0 1 2}$ & $\mathbf{2 0 1 4}$ & $\mathbf{2 0 1 9}$ \\
\hline \multirow{2}{*}{ Nombre total des espèces ligneuses } & 45 & 58 & 49 & 32 & 42 & 43 \\
\cline { 2 - 7 } & 2012 & $\mathbf{3}$ & Site 3 & \multicolumn{3}{|c|}{ Site 4 } \\
\hline Nombre total des espèces ligneuses & 45 & 52 & $\mathbf{2 0 1 9}$ & $\mathbf{2 0 1 2}$ & $\mathbf{2 0 1 4}$ & $\mathbf{2 0 1 9}$ \\
\hline
\end{tabular}

On note que dans l'ensemble, le nombre d'espèces recensées en 2014 était plus qu'en 2012. Les plus fortes différences concernent le site 1 et 2 . Les résultats du recensement des espèces en 2019 montre que seul le site 4 a enregistré une augmentation du nombre d'espèces rencontrées.

3.2 Les espèces ligneuses et biodiversité : Le nombre total d'espèce recensée dans l'ensemble des 4 sites d'étude est de 87 reparti en 56 genres et 22 familles. La famille des Fabaceae domine le peuplement avec 27 espèces recensées. Les familles suivantes: Apocynaceae, Arecaceae, Balanitaceae, Bignonaceae, Cannabaceae, Capparaceae, Chrysobalanaceae, Ebenaceae, Sapotaceae et Ximeniaceae sont présentes avec chacune une seule espèce rencontrée. L'indice de diversité spécifique de Shannon a été estimé pour chaque site et selon l'année (Tableau 5). Le site 1 possède la diversité spécifique la plus élevé. En revanche, le site 3 a la diversité spécifique la plus faible.

Tableau 5: Indice de diversité spécifique de Shannon

\begin{tabular}{|l|c|c|c|}
\hline \multicolumn{1}{|c|}{ Années } & $\mathbf{2 0 1 2}$ & $\mathbf{2 0 1 4}$ & $\mathbf{2 0 1 9}$ \\
\hline Sites 1 & 3,12 & 5,23 & 4,74 \\
\hline Site 2 & 1,66 & 3,97 & 3,12 \\
\hline Site 3 & 1,11 & 1,24 & 1,05 \\
\hline Site 4 & 1,73 & 1,97 & 1,62 \\
\hline
\end{tabular}

3.4 Evolution d'un indicateur de structure : la circonférence à la base : Dans cette étude, seule la circonférence à la base est présentée comme indicateur de structure. Les hauteurs des individus ligneux ont été également mesurées et les traitements statistiques ont permis de noter que des conclusions quasi-identiques pouvaient être tirées. Les figures 3 et 4 montrent l'évolution de la proportion de brin (\%) par site et selon l'année. 


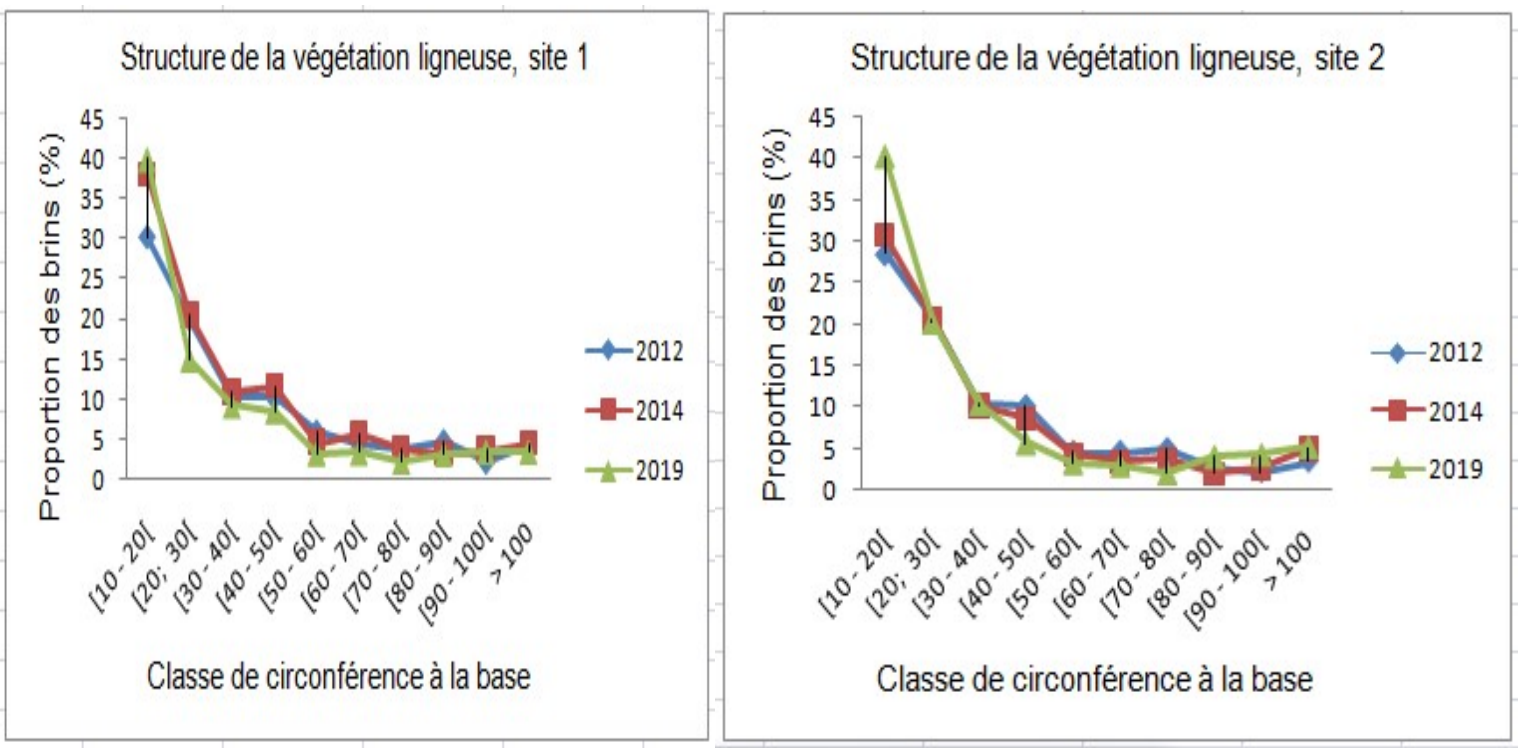

Figure 3 : Evolution de la proportion de brins (\%) par site (1ou 2) et selon l'année.
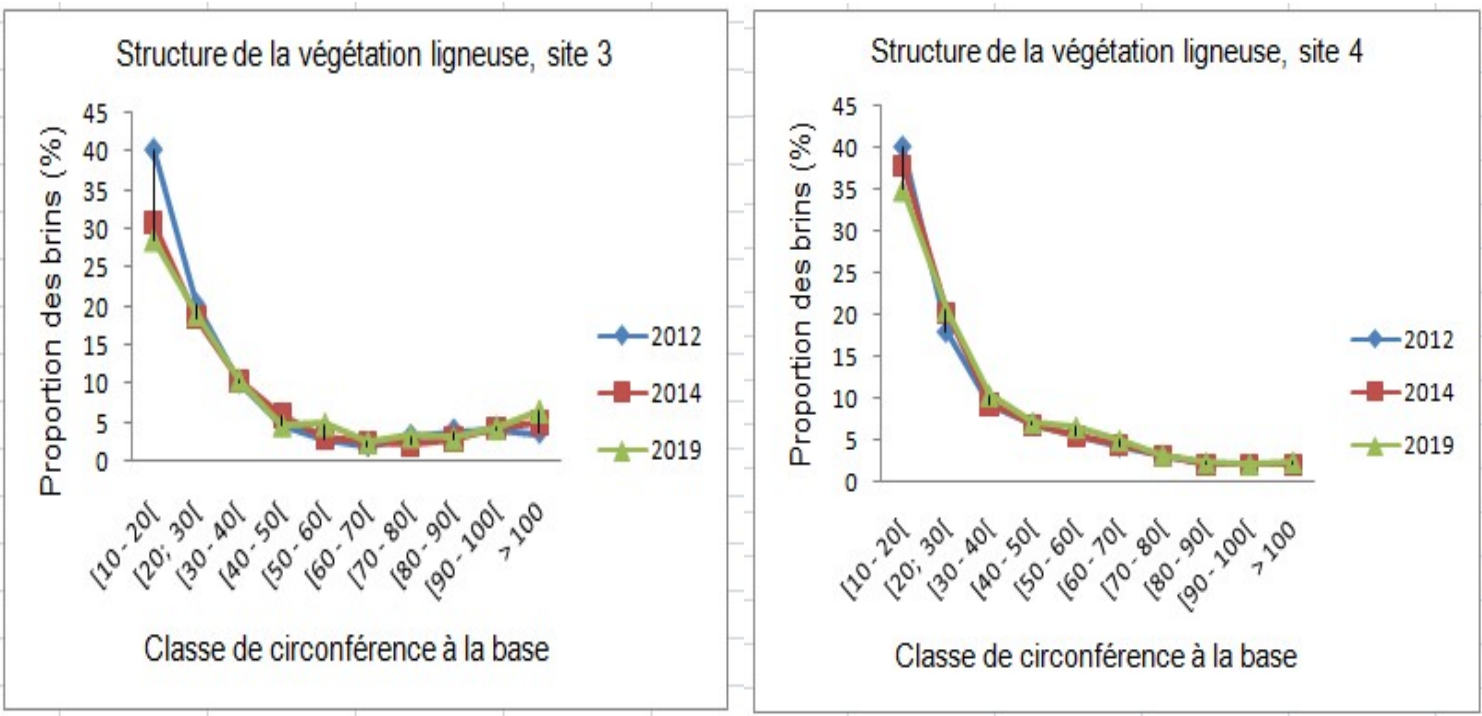

Figure 4 : Evolution de la proportion de brins (\%) par site (3ou 4) et selon l'année.

Les sites 1 et 2 ont des effectifs plus élevés en 2019 comparativement aux années 2014 et 2012.

3.3 Evolution des indicateurs de production

3.3.1 La densité des peuplements ligneux: Les densités moyennes pour l'ensemble des 4 sites expérimentaux et selon l'année sont les suivants : 1517 pieds à l'hectare pour 2012, 2430 pieds à l'hectare pour 2014 et 2304 pieds à l'hectare pour 2019.

3.3.2 La surface terrière: Elle est, en moyenne de $10,12 \mathrm{~m}^{2} /$ ha pour $2012 ; 11,16$ $\mathrm{m}^{2} /$ ha pour l'année 2014 et de $11,65 \mathrm{~m}^{2} / \mathrm{ha}$ pour l'année 2019. L'analyse de l'évolution de la surface terrière à l'hectare met en évidence : une régénération de l'ensemble du peuplement ligneux de Parc National de Manda; la savane arborée claire présente une faible rétraction du couvert végétal et dans l'espace selon les sites et dans le temps (2012, 2014 et 2019), on note de fortes variations de la surface terrière à l'hectare.

3.3.3 Volumes de bois sur pied: Pour l'ensemble des sites expérimentaux, on évalue le 
volume moyen de bois sur pieds à $12,31 \mathrm{~m}^{3} /$ ha en 2012 , à $12,46 \mathrm{~m}^{3} /$ ha en 2014 et $12,08 \mathrm{~m}^{3} /$ ha en 2019. Ces valeurs indiquent que les variations de volume moyen de bois sur pieds sont très faibles pour les sites pris dans leur ensemble.
3.4 Analyse de la dynamique des espèces indicatrices de l'évolution du milieu

3.4.1 Les espèces qui sont dans un processus d'évolution régressive: Densité des espèces situées dans un processus d'évolution régressive est présentée dans le tableau 6.

Tableau 6 : Densité des espèces situées dans un processus d'évolution régressive

\begin{tabular}{|l|l|c|c|c|c|}
\hline $\mathbf{N}^{\mathbf{O}}$ & Espèces & $\mathbf{2 0 1 2}$ & $\mathbf{2 0 1 4}$ & $\mathbf{2 0 1 9}$ & Statut \\
\hline 1 & Afæelia Africana & 3 & 0 & 0 & $\mathrm{R}$ \\
\hline 2 & Amblygonocarpus andongensis & 0 & 1 & 0 & $\mathrm{R}$ \\
\hline 3 & Bombax costatum & 0 & 2 & 0 & $\mathrm{R}$ \\
\hline 4 & Burkea africana & 9 & 7 & 2 & PER \\
\hline 5 & Cordia africana & 0 & 1 & 0 & $\mathrm{R}$ \\
\hline 6 & Hymenocardia acida & 9 & 4 & 1 & PER \\
\hline 7 & Isoberlinia doka & 0 & 3 & 0 & $\mathrm{R}$ \\
\hline 8 & Lonchocarpus laxiflorus & 0 & 1 & 0 & $\mathrm{R}$ \\
\hline 9 & Parinari curatellifolia & 2 & 0 & 1 & $\mathrm{R}$ \\
\hline 10 & Pericopsis laxiflora & 1 & 1 & 1 & $\mathrm{R}$ \\
\hline 11 & Prosopis africana & 0 & 18 & 7 & PER \\
\hline 12 & Pterocarpus lucens & 21 & 7 & 4 & PER \\
\hline 13 & Sclerocarya birrea & 3 & 1 & 0 & PER \\
\hline 14 & Strychnos spinosa & 0 & 1 & 0 & $\mathrm{R}$ \\
\hline 15 & Swartia madagascariensis & 0 & 1 & 0 & $\mathrm{R}$ \\
\hline 16 & Vitex doniana & 5 & 2 & 1 & PER \\
\hline
\end{tabular}

R: Rare

PER: Processus d'évolution régressive

3.4.2 Les espèces qui sont dans un processus d'évolution progressive : De même les espèces qui sont dans un processus d'évolution progressive, ont été caractérisées à base des densités totales des individus de chaque espèce, et de leur présence/absence lors des inventaires successifs. Le tableau 7 présente le statut de 4 espèces ligneuses.

Tableau 7 : Densité des espèces situées dans un processus d'évolution progressive

\begin{tabular}{|l|l|c|c|c|c|}
\hline $\mathbf{N}^{\mathbf{O}}$ & Espèces & $\mathbf{2 0 1 2}$ & $\mathbf{2 0 1 4}$ & $\mathbf{2 0 1 9}$ & Statut \\
\hline 1 & Anogeissus leiocarpa & 351 & 585 & 563 & PEP \\
\hline 2 & Combretum collinum & 310 & 327 & 344 & PEP \\
\hline 3 & Combretum glutinosum & 281 & 239 & 228 & PEP \\
\hline 4 & Detarium microcarpum & 519 & 615 & 698 & PEP \\
\hline
\end{tabular}

PEP : Processus d'évolution progressive

\section{$4 \quad$ DISCUSSION}

4.1 Les communautés végétales: petites tailles peuvent ne plus être observées. Lorsque l'inventaire est effectué après le Globalement, le nombre total de famille selon passage du feu, des familles dont l'ensemble des représentants est composé d'individus de les sites diffère peu. La figure 2 montre que les Fabaceae et les Combretaceae dominent les 
peuplements avec respectivement $31 \%$ et $14 \%$ du nombre total d'espèces rencontrées. En ce qui concerne les Combretaceae, les espèces Combretum collinum et Combretum glutinosum rencontrées en savane et forêt claire sont présentes dans tous les relevés. Elles sont résistante à la sécheresse et aux feux de brousse (Adjonou et al., 2009). On note, Comme dans le cas des familles, les variations observées selon l'année pour chacun des sites pourraient être liées à la période de l'inventaire. Pour rejoindre Faye et al., (2008), après le passage des feux, la totalité des individus de certaines espèces peut être carbonisée. Cette observation est fortement plausible car le pas de temps qui sépare les différentes années d'inventaire semble relativement court pour permettre d'évaluer des «apparitions » ou «disparitions » d'espèces. Cependant, on note que certaines espèces ligneuses affichent des préférences pour des sites: ainsi, Terminalia macroptera, Tamarindus indica, Khaya senegalensis, Ficus thonningii, Combretum glutinosum et Acacia sieberiana semble être inféodés au site 3. D'autres espèces comme Amblygonocarpus andongensis, Afrelia africana, Isoberlinia doka et Vitex doniana sont uniquement rencontrées dans le site 1 . Il ressort des résultats de l'inventaire que, la flore est riche de 87 espèces ligneuses appartenant à 22 familles et 56 genres. Ces familles sont dominées par les Fabaceae, les Combretaceae et les Moraceae. Selon Saradoum (2012), ces familles sont classées parmi les plus importantes de la flore vasculaire du Tchad. Les résultats montrent aussi que le nombre d'espèces recensées en 2019 était plus élevé qu'en 2012, ce qui s'explique par le retour des espèces les plus sensibles à la forte pression anthropique. Ce résultat corrobore, celui trouvé par Sambou (2004), Adjonou et al. (2009) et Badji et al. (2014) qui ont montré, que la mise en défens permet une augmentation du nombre d'espèces ligneuses par rapport à la situation de départ.

4.2 Evolution d'un indicateur de structure : la circonférence à la base : Les caractéristiques structurales sont des indicateurs majeurs pour mesurer l'évolution qualitative et quantitative des peuplements forestiers
(Adjonou et al., 2010). Les figures 3 et 4 montrent que, les sites 1 et 2 ont des effectifs plus élevés en 2019 comparativement en 2014 et en 2012. Une forte régénération s'est effectuée durant l'année 2019 grâce à la mise en œuvre du Projet Conservation et Utilisation Durable de la Biodiversité dans le Moyen Chari. Contrairement aux sites 1 et 2, les site 3 et 4 présentent pour l'année 2019 une forte baisse des effectifs de jeunes plants. Cette différence de densité des peuplements observée pourrait être liée aux caractéristiques écologiques des milieux d'étude, notamment les types de sol, la topographie, le recouvrement. Mais globalement, la structure de la strate ligneuse n’a pas beaucoup évolué en 8 ans. En 2012, le peuplement végétal comportait davantage de vieux arbres surtout Danelia oliveri, Diospyros mespiliformis, Tamarindus indica, Terminalia laxiflorus et Khaya senegalensis. Si les populations de Detarium microcarpum, Annona senegalensis, Piliostigma thonningii, Combretum collinum, Combretum glutinosum et Combretum nigricans sont dans l'ensemble constituées par de petits arbustes, celles de Bombax costatum, Khaya senegalensis, Ficus platyphylla sont formées au contraire par une majorité d'arbres dont la circonférence mesure plus de $80 \mathrm{~cm}$. Le suivi des indicateurs de structure de 2012 et 2019 n'a pas permis de déceler d'importantes variations dans l'espace et dans le temps. Les seules variations notées concernent la population de jeunes plants qui est très sensible aux changements climatiques et aux feux de brousse.

\subsection{La densité des peuplements} ligneux : La densité moyenne a donc connu un accroissement entre 2012 et 2014 et affiche à partir de 2014 une certaine stabilité. Les sites 1, 2 et 3 présentent un comportement tout à fait particulier: tout se passe comme si pendant 2 ans (2012 - 2014) la régénération a été importante et la mortalité précoce insignifiante. L'examen du tableau VI, permet de noter que nous trouvons à côté d'une population de jeunes plants, peu d'arbres âgés. De 2014 à 2019, les densités totales du site 1, 2 et 3 ont connu une baisse avec des facteurs régressifs 
respectivement de 0,92 , de 0,71 et 0,8 . Les faibles valeurs de densité obtenues peuvent s'expliquer par la forte anthropisation pour le bois de chauffe et de fourrage qu'a subi cette aire protégée. Concernant le site 4 , les densités totales entre 2012 et 2014 n'ont pratiquement pas variées. Cependant, on note une forte augmentation de cette densité pour la période allant de 2014 à 2019. Le comportement de ce site, laisse supposer une grande espérance de vie. Le peuplement du site 4 apparaît assez proche de l'équilibre. Il faut noter que contrairement aux autres sites, le site 4 présente pour la période 2014-2019, une augmentation significative de la densité totale des brins appartenant aux classes de circonférence suivantes : $40-50 \mathrm{~cm} ; 60-70 \mathrm{~cm}$ et $70-80 \mathrm{~cm}$. La densité moyenne entre 2012 et 2014 s'est accrue et à partir de 2014 elle s'est stabilisée. En fait, il s'agit d'une population de jeunes plants avec peu d'arbres âgés disséminés.

4.4 La surface terrière : Pour l'ensemble, la surface terrière par ha n'est pas faible et a connu une augmentation graduelle de 2012 à 2019. Les surfaces terrières par hectare sont plus élevées pour le site 3. En revanche, les valeurs les plus faibles sont notées pour le site 2. Pour le site 1 , les surfaces terrières à l'hectare sont restées stables entre 2012 et 2014 malgré la forte augmentation de la densité totale. Les sites 2,3 et 4 présente une légère augmentation de la surface terrière à l'hectare entre 2012 et 2014, mais pendant le même laps de temps, l'effectif global de brins est multiplié par 6 pour le site 2 ; par 1,7 pour le site 3 et par 0,8 pour le site 4 . L'examen de la répartition des surfaces terrières en fonction des sites et selon l'année permet d'avoir des conclusions plus accusées de l'évolution dans le temps des peuplements ligneux. Dans le temps, la mortalité des gros brins est faible. Ainsi, tout effort de gestion des peuplements doit faire un choix prioritaire de contrôle des jeunes plants et des petits brins.

4.5 Volumes de bois sur pied : La comparaison entre site fait ressortir que le site 4 présente les volumes de bois sur pieds les plus élevés. En plus, ce site exhibe les plus petites différences selon les années malgré une très faible augmentation graduelle entre 2012, 2014 et 2019. Les volumes de bois sur pieds les plus faibles sont notés dans le site 2 malgré une densité totale plus élevée que le site 3 . Ces résultats soulignent que le site 3 , héberge une importante population d'arbres âgés. Concernant le site 1, le volume de bois sur pieds présente des valeurs quasi-identiques pour 2012 et 2014. On note une valeur légèrement supérieure pour l'année 2014. Cette valeur supérieure du volume sur pieds notée en 2014 est probablement à mettre en relation avec l'augmentation des densités totales des brins à l'hectare observée en 2014 pour les classes de circonférences suivantes : classe $[50-60[\mathrm{~cm}$ et classe $[60-70[\mathrm{~cm}$. Les résultats montrent que les variations de volume moyen de bois sur pieds sont très faibles pour les sites pris dans l'ensemble. Même si à l'intérieur d'un même site les variations dans le temps sont quasiinsignifiantes, on note toutefois, des différences importantes entre sites.

4.6 Analyse de la dynamique des espèces indicatrices de l'évolution du milieu : La caractérisation des espèces qui sont dans un processus d'évolution régressive est faite sur la base des densités totales des individus de chaque espèce, et de leur présence / absence lors des inventaires successifs. L'analyse des résultats obtenus permet de générer le tableau VI. Six espèces se situent dans un processus d'évolution régressive; Il s'agit de: Burkea africana, Hymenocardia acida, Prosopis Africana, Pterocarpus lucens, Sclerocarya birrea et Vitex doniana. Dix autres espèces peuvent être qualifiées de rares. Ce sont Afzelia Africana, Amblygonocarpus andongensis, Bombax costatum, Isoberlinia doka, Cordia africana, Strychnos spinosa, Parinari curatellifolia, Swartzia madagascariensis, Pericopsis laxiflora et Lonchocarpus laxiflorus. L'état menacé ou disparu de certaines espèces s'explique surtout par la pression exacerbée sur ces dernières du fait de leurs multiples usages mais aussi de l'absence de leur régénération. Cela peut être lié à l'élevage ou aux feux répétitifs dans la localité. Sounon et al., 2007 et Melom et al. (2015) notent que la structure et l'équilibre des savanes et des forêts 
sèches sont marqués par les perturbations anthropiques telles que le feu ou l'élevage. En effet, les premières sur cette liste d'espèces menacées (Afzelia Africana, Amblygonocarpus andongensis, Bombax costatum Isoberlinia doka, Cordia africana, Strychnos spinosa, Parinari curatellifolia, Swartiia madagascariensis, Pericopsis laxiflora et Lonchocarpus laxiflorus) sont utilisées aussi bien dans l'alimentation des hommes et des animaux, la pharmacopée traditionnelle, le bois d'énergie et d'artisanat, mais leur régénération est fortement atteinte. L'absence d'un nombre important de jeunes plants notée chez Acacia senegal serait liée à l'utilisation des graines et des fruits dans l'alimentation du bétail (Binot et al., 2007). À cela s'ajoute la sélection des jeunes plants par le pâturage et l'influence du broutage sur le taux de croissance des jeunes arbres (Richad et al., 2014). Mais dans l'ensemble des sites, on constate une forte régénération. À l'échelle des sites, les espèces qui se multiplient par semis (Combretum glutinosum, Acacia. seyal, Faidherbia albida) sont plus favorisées par la mise en défens que celles qui se multiplient par rejet de souches (Anogeissus leiocarpus, Terminalia laxiflora, Detarium microcarpum). Selon Melom et al. (2015), la capacité à produire des rejets à partir de la base de la tige ou de racines dépend de plusieurs facteurs dont entre autres un stress (labour, feu...) et peut généralement être induite par les perturbations anthropiques. Ce qui n'est pas le cas dans les aires protégées où les coupes sont interdites. Deux espèces ont un fort impact sur le milieu, il s'agit Detarium microcarpum (densité 597 individus à l'hectare) et Terminalia laxiflora (densité 533 individus à l'hectare). Detarium microcarpum a une capacité de régénération très élevée. Ce résultat corrobore celui d'Agbo et al., (2018) qui ont travaillé sur la dynamique SpatioTemporelle d'habitats convenables pour

\section{CONCLUSION}

L'évolution du peuplement ligneux du PNM diffère selon le site. La dégradation affecte particulièrement les formations végétales situées à proximité des villages. Dans le temps, le suivi des indicateurs de structure conduit en 8 ans
Detarium microcarpum au Bénin ont montré que cette espèce est envahissante. Sa multiplication végétative naturelle se fait par rejet et par drageon. Le Terminalia laxiflora est une espèce très disséminée. Sa multiplication végétative naturelle se fait également par rejet et par drageon. Trois espèces ont un impact assez fort sur le milieu; il s'agit de Annona senegalensis (densité de 312 individus par hectare), Anogeissus leiocarpa (densité de 322 individus par hectare) et Piliostigma thonningii (densité de 323 individus par hectare). Parmi ces trois espèces, seule Piliostigma thonningii se multiplie naturellement en dehors de rejet de souche par semis. Annona senegalensis se multiplie naturellement par drageon et Anogeissus leiocarpa par pousse adventive et rejet de collet. Les espèces suivantes ont un impact perceptible sur le milieu. Il s'agit: Combretum collinum, Diospyros mespiliformis, Combretum glutinosum, Entada africana, Daniellia oliveri, Dichrostachys cinerea, Ximenia americana, Combretum nigricans, Combretum paniculatum, Hexalobus monopetalus, Gardenia ternifolia, Grewia bicolor. L'ensemble de ces différentes espèces a une multiplication végétative naturelle par rejet sauf: Gardenia ternifolia et Ximenia americana. Les deux espèces suivantes ont en plus des rejets comme multiplication végétative naturelle, le drageonnage: Daniellia oliveri et Dichrostachys cinerea. Cette situation nécessite l'application imminente de nouvelles techniques de gestion. Cela devra nécessairement prendre en compte l'histoire de l'évolution du milieu avec toutes ses composantes (conditions édaphoclimatiques, la flore, les pratiques locales, la transhumance, etc.) mais surtout passera par une meilleure promotion de la régénération naturelle assistée et qui se révèle être un succès avec la participation effective des autochtones.

témoigne de la variabilité inter-annuelle des pluies. Le potentiel productif du milieu subit de profondes modifications d'une année à l'autre. Les densités totales peuvent osciller dans le rapport de 1 à 7 en fonction des aléas 
climatiques. Si pour un même site, nous n'avons pas pu montrer de différence significative quant à la structure du peuplement en circonférence à la base, l'étude conduite se justifie dès lors que sont abordés les problèmes de régénération. La composition qualitative et quantitative des populations dépend du type de formation végétale choisi. Dans l'ensemble des sites expérimentaux, l'abondance des ligneux est importante en 2012 qu'en 2014. Ce phénomène présente un maximum d'ampleur sur le site 2 où le degré d'abondance a augmenté de 7,5 en 2 ans. Cette double variabilité spatiale et temporelle, constatée et analysée nous amène à être prudent dès qu'il s'agit de généraliser les résultats obtenus à l'ensemble de l'écosystème du PNM qui recouvre des zones diverses. Il est donc

\section{REMERCIEMENTS}

Les auteurs expriment leur profonde gratitude aux personnes qui les ont aidés dans la réalisation de cet article, en particulier le Chef

\section{RÉFÉRENCES BIBLIOGRAPHIQUES}

Adjonou K, Bellefontaine R. et Kokou K: 2009. Les forêts claires du Parc National Oti Kéran au Nord Togo : structure, dynamique et impacts des modifications climatiques récentes. Sécheresse, 1 (5) : 1 10.

Adjonou K, Djiwa O, Kombate Y, Kokutse AD. et Kokou K: 2010. Étude de la dynamique spatiale et structure des forêts denses sèches reliques du Togo : implications pour, une gestion durable des aires protégées. International Journal of Biological and Chemical Sciences, 4 (1): 168183.

Agbo RI, Idohou R, Vihotogbé R, Antoine Abel Missihoun A, Dagba RA, Assogbadjo AE. et Agbangla C: 2018. Spatio-temporal dynamics of suitable habitats for Detarium microcarpum Guill. \& Perr. (Caesalpiniaceae), a priority food tree species in Benin (West Africa). Modeling Earth Systems and Environment, 1:1-10. nécessaire d'augmenter le nombre de sites de suivi afin de prendre en compte les divers facteurs environnementaux pour une bonne généralisation des résultats. Cette région naturelle, essentiellement composée de milieu savanicole, avec quelques massifs forestiers très localisés, ne représente pas une originalité bioécologique remarquable. Sa flore et sa faune sont celles de bien d'autres régions d'Afrique soudanienne. Mais son originalité actuelle est dû au fait que, les résultats de ce travail permettent de sauvegarder les arbres qui sont devenus rares ou situés dans un processus d'évolution régressive en zone soudanienne. Aussi, les résultats quantitatifs permettent de suivre l'évolution et le développement du peuplement ligneux, et les variations de la biomasse végétale au cours du temps.

de canton de Niellim et les responsables des Eaux et Forêt du Parc National de Manda.

Badji M, Sanogo D. et Akpo LE : 2014. Dynamique de la végétation ligneuse des espaces sylvo-pastoraux villageois mis en défens dans le Sud du Bassin arachidier au Sénégal. Bois et Forêts des Tropiques, 319 (1) : 43-52.

Ballah SR : 2008. Le Parc national de Manda: dynamique des ressources naturelles et cogestion, mémoire de DEA, Université de Yaoundé1, Cameroun, 83 pp.

Binot A, Hanon L, Ndotam TI. et Daou VJ : 2007. Aménagement de territoires multi usages en périphérie d'une aire protégée africaine: entre enjeu de conservation et développement; le cas du Parc National de Zakouma (sud-est du Tchad). GEPAC-ULB, Bruxelles, 7-52.

Chardonnet B. et Boulanodji E: 2010. Plan d'aménagement du Parc National de Manda de 2011 à 2021. Rapport provisoire. République du Tchad, Ministère de l'Environnement et des 
Ressources Halieutiques /DPNRFC, 175 pp.

Faye E, Diatta M, Samba A NS. et Lejoly J : 2008. Usages et dynamique de la flore ligneuse dans le terroir villageois de Latmingué (Sénégal). Journal des Sciences et Technologies, $7: 43-58$.

Kolmagne MN : 2000. Etude du peuplement de faune et de flore dans le parc national de Manda. Etats de surface : - Transects dans le parc, - Elément de typologie des espèces inventoriées, - Axes d'intervention comme suite à cette étude. Rapport consultatif. APRODEPIT, CARE-TCHAD. Ndjamena. 72 pp.

Madjimbe G, Goalbaye T, Mamounata OB. et Ngarikla B: 2018. Evaluation des ressources ligneuses et leur exploitation comme bois de chauffe et de service dans le Département de Barh-kôh au sud du Tchad. International Journal of Biological and Chemical Sciences, 12 (6): 2856-2870.

Melom S, Mbayngone E, Béchir AB, Ratnab N. et Mapongmetsem PM: 2015. Caractéristiques floristique et écologique des formations végétales de Massenya au Tchad (Afrique centrale). Journal of animal \& Plan Sciences, 2015. Vol. 25, Issue 1. 3799-3813.

Minda MS, Ousmane N, Mariama DD, Saradoum G, Ally D. et Aliou G: 2015. Caractérisation des peuplements ligneux sur le tracé de la Grande Muraille Verte au Tchad. International Journal of Biological and Chemical Sciences, 9(5): 2617-2627.

Ouya B: 2005. Les enjeux autour des aires protégées du sud-est du Tchad, dynamique d'occupation et partage de l'espace: cas du Parc National de Manda et Zakouma. Mémoire de Master I, Université de Montpellier III. 95pp.

Pias J : 1964. Les sols du Tchad. VIIIème Congrès International de la Science du Sol, Bucarest-Roumanie. Comptes rendus, Vol V, 145-151.
Rachad KF M, Ali J, Odjoubere A. et Brice S: 2014. Caractérisation floristique et analyse des formes de pression sur les forêts sacrées ou communautaires de la Basse Vallée de l'Ouémé au Sud-Est du Bénin. Afrique Science 10(2) (2014) 243 257.

Sambou B : 2004. Évaluation de l'état, de la dynamique et des tendances évolutives de la flore et de la végétation ligneuse dans les domaines soudanien et subguinéen du Sénégal. Thèse de Doctorat d'État. Université Cheikh Anta DIOP de Dakar, Institut des Sciences de l'Environnement, $210 \mathrm{pp}$.

Saradoum G : 2012. Etude phytosociologique et diagnostic faunique du Parc National de Manda au Tchad ; éléments pour un aménagement. Thèse de Doctorat, Université Cheikh Anta Diop de Dakar, Dakar, 183 pp.

Sarr O, Diatta AS, Gueye M, Ndiaye PM, Guisse A. et Akpo LE : 2013. Importance des ligneux fourragers dans un système agropastoral au Sénégal (Afrique de l'ouest). Revue Méd. Vét.Toulouse, 2013, 164, 1, 2-8.

Sounon Bouko B, Sinsin B. et Goura Soulé B : 2007. Effets de la dynamique d'occupation du sol sur la structure et la diversité floristique des forêts claires et savanes au Bénin. Tropicultura, 25 (4) : 221-227.

Tchago B: 1999. Les systèmes de gestion participative rationnels avec une exploitation et une gestion optimale des ressources par l'atténuation des pressions qui existent dans et autour du Parc National de Manda. Rapport de consultation, Tchad, $108 \mathrm{pp}$.

UICN/PACO : 2008. Évaluation de l'efficacité de la gestion des aires protégées: aires protégées du Tchad. $47 \mathrm{pp}$. 